INV :TED PAPER: XVI INTERNATIONAL CONFERENCE ON THE PHYSICS OF ELECTRONIC AND ATOMIC COLLISIONS, JULY 20 - ALIGUST 1, 1989, NEW YORK, NEW YORK

CONF-890708--1

DE89 015908

\title{
PHOTODETACHENT COLLISIONS
}

David J. Pegg

Department of Physics, University of Tennessee, Knoxville, Tennessee 37996-1200, USA and

Physics Division, Oak Ridge National Laboratory, Dak Ridge, Tennessee 37831-6377, USA

\begin{abstract}
A crossed-beams apparatus has been used to measure the cross sections (angular differential and integral) for photodetaching electrons from metas:able $\mathrm{He}^{-}$and stable $\mathrm{B}^{-}$Ions at $\lambda=696.2 \mathrm{~nm}$. Kinematic effects were exploited, in a novel way, to enhance the precision of the measurements. The partial (angular integral) cross sections for $\mathrm{He}^{-}$ photodetachment are $\sigma\left(2^{3} \mathrm{~S}\right)=22.9 \pm 1.0 \mathrm{Mb}$ and $\sigma\left(2^{3} \mathrm{P}\right)=10.0 \pm 0.6 \mathrm{Mb}$. The corresponding cross section for $B^{-}$is $\sigma\left(2^{2} \mathrm{P}\right)=16.5 \pm 2.1 \mathrm{Mb}$. The calculated cross section for the photodetachment of $\mathrm{D}^{-}$was used to estabilsh an absolute scale for the relative measurements. Radiative attachment cross sections are derived from the photodetachment results using the principle of detalled balance.
\end{abstract}

\section{INTRODUCTION}

Interest in photon-ion interactions has grown rapldly over the past few decades, due primarily to the advent of lasers and synchrotron light sources. At the Brighton ICPEAC, for example, Dolder reported on elegant experiments designed to measure cross sections for photolonization of positive atonic lons. In this progress report, I shall at tempt to complement that contribution by describing measurements of cross sections for the photodetachment of negative atomic ions made recently at the Oak Ridge National Laboratory.

The ploneering work on photodetachment began at NBS in the 1950's and early 1960's with the development, by Branscomb and Fite, ${ }^{2}$ of a crossed photon-negative ion beams apparatus. In these early experiments, an arc lamp was used as the light source. The absolute cross sections that were measured have been reviewed by Branscomb and Smlth. ${ }^{3}$ A second generation of crossed-beams experiments began at JILA in the late 1960's and $1970^{\prime} \mathrm{s}$, when the conventional light source was replaced by a laser. Th1s modification not only greatly enhanced available photon fluxes but also made it possible, for the first time, to measure photoelectron angular distributions with relative ease. Most of the experiments of this period were designed primarily, however, to measure electron affinities and determine relative changes in cross sections, particularly in the vicinities of thrisholds and resonances. This work has been reviewed by Lineberger. 5 Somewhat suprisingly perhaps, only a few absolute photodetachment cross section measurements have been re. "rted to date. Photodetachuent cross sections are not only of intrinsic interest but they frequently form the most accesible path to the determination of cross sections for the far less probable process of radiative attachment.

The present paper describes the use of energy- and angle-resolved photoelectron detachment spectroscopy to determine partial cross sections (angular differential and integral) for the photodetachment of the wetastable HeIon at a wavelength of $\lambda=696.2 \mathrm{~nm}$. Brehm et al.6 reported the f1rst energy-resolved studies of the photodetachment of $\mathrm{He}^{-}$. In this experiment, a crossed-beams technique, which employed a flxed-frequency laser, was used to determine the electron affinity of He( $\left(2^{3} \mathrm{~s}\right)$. As a by-product of these measurements, the Invest1gators were also able to estimate, within a factor of two, the cross sectlons for photophotodetaching $\mathrm{He}^{-}$at $\lambda=514.5 \mathrm{~nm}$. The large quoted uncertainties reflect the fact that no information on the photoelectron angular distributions was obtained in the experiment. Total cross sections for the photodetachment of $\mathrm{He}^{-}$ (via two competing unresolved exit channels) have been reported by compton ot $a 1.7$ and Hoden et al. The croas ection for phota-

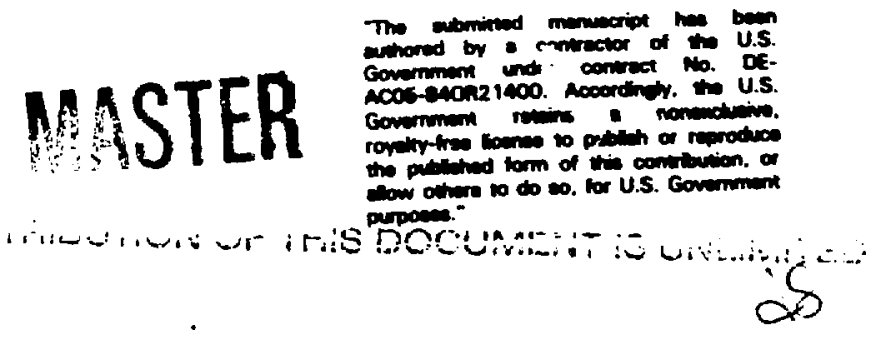


detachment of $B^{-}$at $\lambda=696.2 \mathrm{~nm}$ has also been measured in the present work for the first time.

\section{THEORETICAL CONSIDERATIONS}

The $\mathrm{He}^{-}$ion is unstable as a result of being formed in the spin-aligned (1s2s2p) ${ }^{4} \mathrm{P}$ state (see Fig. 1) when an electron is captured by an excited He $\left(1 s 2 s^{3} s\right)$ atom. The quartet state, unable to decay via allowed radiative or autodetachment processes, autodetaches via forbidden processes induced by the relatively weak spindependent interactions. The varying strengths of these interactions result in a differential metastability among the fine structure levels. Lifetimes of the $J=1 / 2,3 / 2$, and $5 / 2$ levels have been measured ${ }^{9}$ to be 10,16 , and $500 \mu \mathrm{sec}$, respectively. Metastable $\mathrm{He}^{-}$Ions are therefore sufficlently long lived that they undergo very litele exponential depletion by autodetachment prior to photodetachment in the present crossedbeams apparatus. Small corrections ( $2 \%$ ) to the measured ylelds are, however, made to account for the unstable nature of this ton.

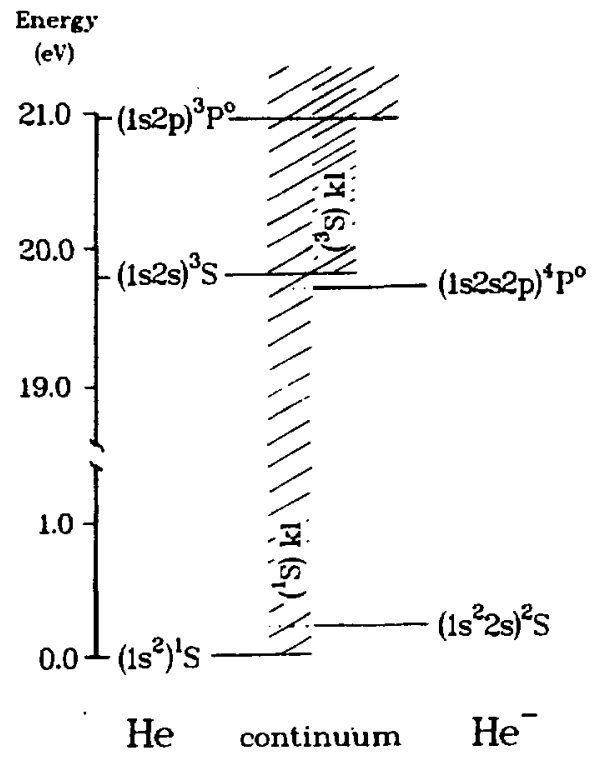

FIG. 1. Partial energy level diagram for the $\mathrm{He}$ and $\mathrm{He}^{-}$systems. The $\mathrm{He}^{-}$ion exists in the metastable $(1 s 2 s 2 p)^{4} P$ state. The $\left(1 s^{2} 2 s\right)^{2} S$ ground state is unbound.

When the $\mathrm{He}^{-}\left(2^{4} \mathrm{P}\right)$ Ion interacts with visible radiation, two competing photodetachment channels to the continuum are open:

$$
\begin{aligned}
& h \nu+H e^{-}\left(2^{4} P\right)+H e\left(2^{3} s\right)+e^{-}(k s, d) \\
& h \nu+H e^{-}\left(2^{4} P\right)+H e\left(2^{3} P\right)+e^{-}(k p) .
\end{aligned}
$$

In this paper, photodetachment processes will frequently be labeled by their exit channels. In an Independent electron description of process (1), a p-orbical electron is ejected, resulting in an outgoing electron represented by s- and d-waves. In process (2), a s-orbital electron is efected, leading to a pure outgoing $p$-wave in the independent electron model. The spectral dependences of the asymetry parameters for both of these processes have recently been measured by Thompson et al.10 Correlation effects are found to play a significant role in determining the shape of the electron emission patterns following photodetachment.

The $\mathrm{B}^{-}$ion is stable, being formed 10 the $\left(1 s^{2} 2 s^{2} 2 p^{2}\right)^{3} p$ state when an electron is captured by a ground state $B\left({ }^{2} P\right)$ atom. At visible wavelengths, only one photodetachment channel is open when $B^{-}\left(2^{3} P\right)$ interacts with radiation

$$
h \nu+B^{-}\left(2^{3} P\right)+B\left(2^{2} P\right)+e^{-}(k s, d) .
$$

Since a p-orbital electron is efected, the outgoing electron is characterized by $s^{-}$and $d-$ partial waves in the independent electron model.

In the present work, the part1al cross sections, $\sigma\left({ }^{3} S\right)$ and $\sigma\left({ }^{3} P\right)$, for the photodetachment processes shown in Eqns. (1) and (2) have each been measured relative to the crosa section, $\sigma\left({ }^{2} \mathrm{~S}\right)$, for the photodetachment of $\mathrm{D}^{-}\left(1^{1} \mathrm{~S}\right)$ lons via the process

$$
h v+D^{-}\left(1^{1} s\right)+D\left(1^{2} s\right)+e^{-}(k p) .
$$

In this process, the emission is well described by an independent electron model and the photoelectron is represented by a pure p-wave resulting in a $\cos ^{2} \theta$ dietribution.

\section{EXPERIMENAI CONSIDERATIONS}

The technique of energy- and angle-resolved photoelectron spectroscopy has been used to investigate the Interaction between negative ions and radiation. Cross sections for photodetachment are determined from measurements of the yields and angular distributions of photoelectrons ejected from the lons at the interaction of crossed laser and negative ion beams. Fig. 2 is a schematic of the crossed-beams apparatus. A fast moving $\left(v \sim 10^{8} \mathrm{~cm} / \mathrm{sec}\right)$ and tenous beam $\left(p \sim 10^{3}\right.$ ions $\left./ \mathrm{cm}^{3}\right)$ of negat1ve lons is 1ntersected perpendicularly by an energy-resolved and linearly-polarized beam of photons from a pulsed dye laser. As a result of the photon-ion interaction, precisely known amounts of energy and angular momentum are transferred to the lon. Following photodetachment events, electrons ejected from the field-free interaction region in the direction of motion of the ion bean (forward-directed electrone) are collected, energy analyzed, and detacted. The angular dis-

\section{DISCLAIMER}

This report was prepared as an account of work sponsored by an agency of the United States Government. Neither the United States Government nor any agency thereof, nor any of their employees, makes any warranty, express or implied, or assumes any legal liability or responsibility for the accuracy, completeness, or usefulness of any information, apparatus, product, or process disclosed, or represents that its use would not infringe privately owned rights. Reference herein to any specific commercial product, process, or service by trade name, trademark, manufacturer, or otherwise does not necessarily constitute or imply its endorsement, recommendation, or favoring by the United States Government or any agency thereof. The views and opinions of authors expressed herein do not necessarily state or reflect those of the United States Government or any agency thereof. 
tribution of the detached photoelectrons can be determined by measuring their yleld as a function of the angle between the electric field vector of the linearly polarized laser beam and the collection direction. This is achieved in the present apparatus by keeping the collection fixed in the forward direction and rotating the laser polarization vector, using a $\lambda / 2$ phase retarder (double Fresnel rhomb). Fig. 3 shows the result of an angular distribution measurement. A typical photoelectron spectrum, taken in this case with the polarization vector parallel to the collection direction, is shown In Fig. 4.

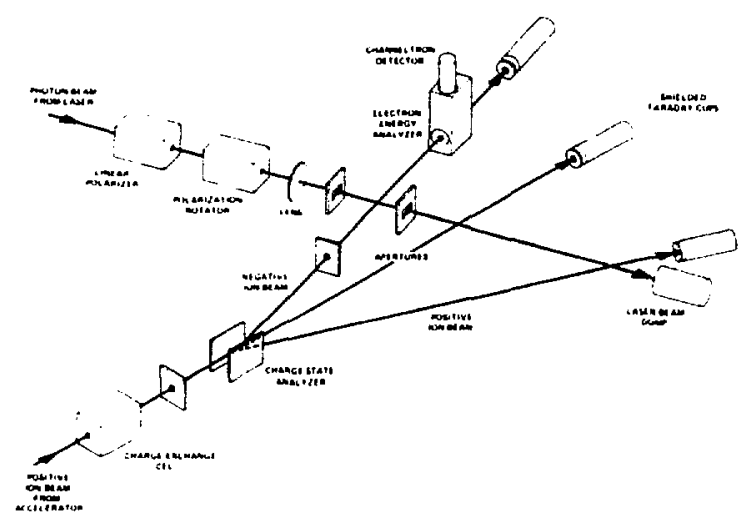

FIG. 2, A schematic view of the crossed laserIon beams apparatus used in the energy- and angle-resolved photoelectron spectroscopy measurements.

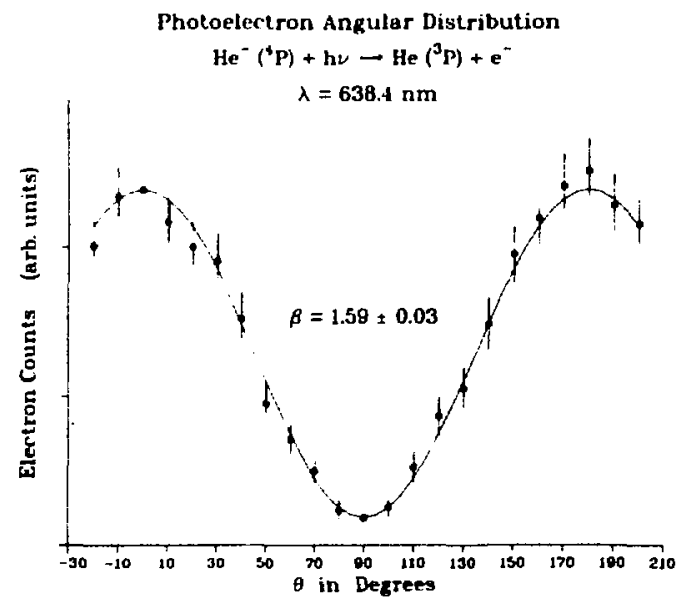

FIG. 3. Angular distribution of electrons photodetached from a $40 \mathrm{keV}$ beam of $\mathrm{He}^{-}$Ion at $\lambda=638.4 \mathrm{~mm}$.

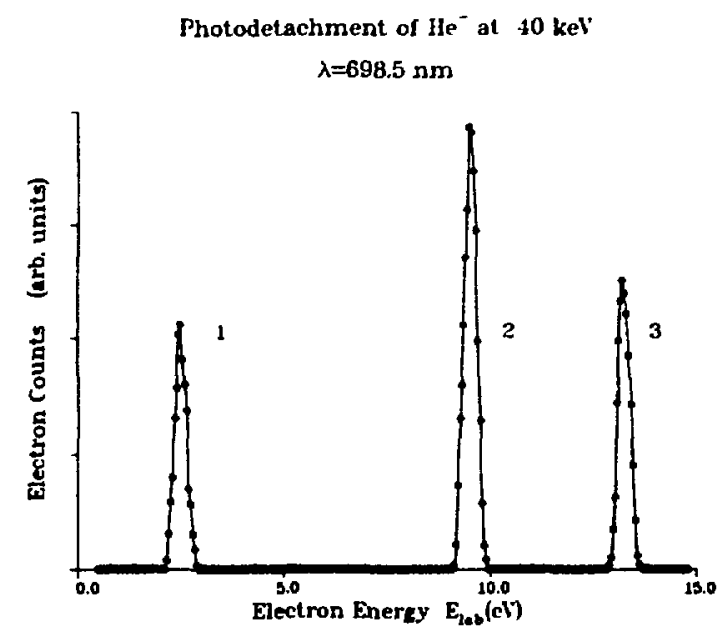

FIG. 4. Energy spectrum of electrons photodetached frcm a $40 \mathrm{keV}$ beam of $\mathrm{He}^{-}$lons at $\lambda=689.5 \mathrm{~nm}$.

The beam that is the source of negative Ions in these measurements is produced by double charge transfer (sequential electron capture) when a momentum-selected positive lon beam from an accelerator is passed through a $L 1$ vapor charge-exchange cell, After a delay of a few microseconds, the beam exiting the cell is charge state analyzed. The negative ion component $\left(S_{1} z\right)$ is then deflected by $10^{\circ}$ into a beam line containing an electron spectrometer consisting of a spherical-sector energy analyzer and a channel-electron multiplier detector. The analyzer is operated in the fixed pass energy, preacceleration mode. About $2 \mathrm{~cm}$ in front of the entrance to the electron spectrometer, the lon beam 18 crossed with the laser beam. Sets of apertures are used to ensure that the overlap of the two beams remains unaltered during the measurements. The flashlamp-pumped pulsed dye laser used in this investigation has a maximum repetition rate of $10 \mathrm{~Hz}$ and a pulse duration of $2.2 \mathrm{ksec}$. The operating laser bandwidth was $0.2 \mathrm{~nm}$. The output power of the laser was carefully chosen to avold saturation on each of the photodetachment processes studied. A $10-\mathrm{cm}$ focal length infection lens focussed the laser beam onto the lon beam in the interaction region. The whole apparatus is surrounded by three mutually orthogonal sets of Helmholtz colls designed to reduce the effect of stray magnetic fields on the electron trajectories.

The data is stored in a CAMAC-based multichannel analyzer data acquisition system. A synchronous detection schewe ts used to extract the photoelectron ignal frow a rathar large

\section{REPRODULED FROM BEST AVAII.ABLE COPY}


electron background. The time structure of the detection scheme is illustrated in Fig. 5. Four multichannel scalers (MCS) are used to record the data. MCSl is triggered by the laser pulse via a fast photodiode detector. It is gated on for 4 usec, after a 1 usec delay, to record the electron signal associated with the laser being on. The delay is set to allow for the finite transit time of electrons through the spectrometer and to avoid noise assoclated with the "firing" of the laser. MCS2, which is operated with the same gate width as MCS1, is triggered on $2 \mu \mathrm{sec}$ after MCS1 is turned of $f$. The purpose of MCS2 is to record, with the laser of $f$, the background associated with electrons generated by lon 1mpact with apertures and, to a lesser extent, electrons produced by detachment followIng collisions between the fons and the residual gas. In the case of metastable lons, a small contribution arises from autodetachment. Subtraction of the contents of MCS1 and MCS2 allows one to effectively discriminate against the background contributions. MCS3 and MCS4 are used to monitor the ion and laser beam intensities for normalization purposes. The lon beam current is collected and integrated in a Faraday cup situated directly downstream of the electron spectrometer. The ion beam intensities were chosen so that approximately the same background of electrons was always incldent on the detector. This made it less likely that pulse pileup effects and count rate-dependent gain changes would affect the relative measurements. The intensity of the photon beam is recorded by monitoring the light scattered from the front face of the infection lens with a photodiode detector. Further detalls of the apparatus can be found in the paper by Pegg et al. 11

As a consequence of the fast moving and unidirectional nature of the source, the kinetic

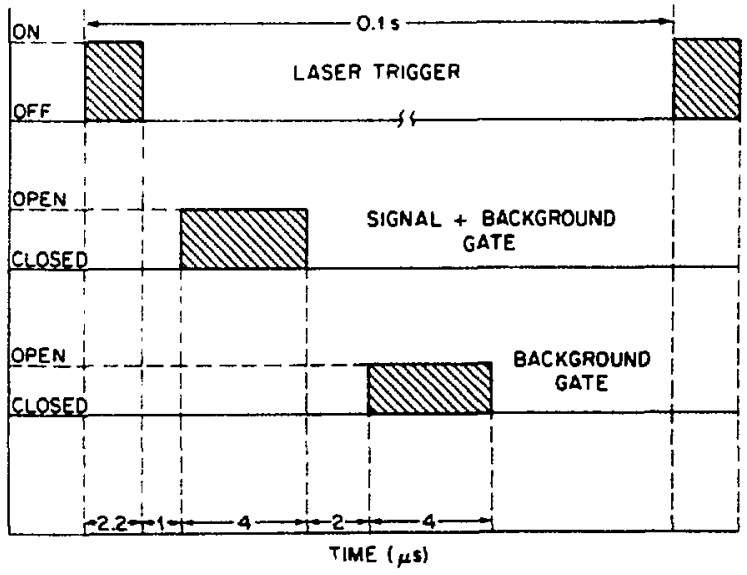

FIG. 5. The time gicructure of the synchronous detection scheme. energles, ylelds, energy distributions, and angular distributions of the photoelectrons efected from the moving lons will be modifled by kinematics. The combination of high-energy beams and forward-directed collection used in the present experiment permits one to effective1y exploit kinematic effects such as spectral peak shifting and doubling. Previous cross section measurements using crossed beams have employed ton beam energies an order of magnitude lower than those used in the present work and collection has been perpendicular to the ion beam direction. The kinematically-shifted energy of a forward-directed $\left(\theta_{L}=0\right)$ electron in the laboratory frame, $E_{\mathrm{L}}$, is related to its energy in the ion frame, $E_{c}$, via the relationship $E_{L}=\mathbb{E E}_{c}$, where $M$ represents a kinematic "magnification" factor, $M=\left(2 \pm \sqrt{\varepsilon / E_{c}}\right)^{2}$. Here, $\varepsilon$ is the energy of an electron moving with the same velocity as the lons of the beam. The positive sign in the factor $M$ corresponds to emission of electrons in the forward direction $\left(\theta_{c}=0\right)$ in the Ion frame and the negative sign corresponds to backward-directed $\left(\theta_{c}=\pi\right)$ emission in the ion frame. If the lon beam velocity exceeds the electron velocity in the Ion frame, both the forward and backward electrons w11l be swept forward in the laboratory frame. Kinematic peak doubling arises since $E_{L}$ becomes double valued in this case. The separation of the twin peaks $18 \Delta \mathrm{E}_{\mathrm{L}}=4 \sqrt{\varepsilon \mathrm{E}_{\mathrm{c}}}$. Details of these and other kinematic modifications have been discussed previously by Pegg et al.12

\section{RELATIVE CROSS SECTIONS}

The angular integral cross section, $\sigma(x)$, for photodetachment of an ion via an exit channel labeled $x$ can be expressed in terms of the yield of photoelectrons, $Y(x)$, ejected in the direction of the linear polarization vector of the light beam in the following manner:

$$
\sigma(x)=\frac{4 \pi Y(x)}{[1+\beta(x)] g(x) \phi(x) k(x) \rho(x) V \Delta Q G T} \cdot
$$

In this expression, $\beta$ is the asymmetry parameter characterizing the shape of the emission pattern, $g$ is the frame-transformed solid-angle ratio, $k$ represents the photoelectron collection and detection efficiency, $p$ is the ion beam density, - is the photon flux, $V$ is the interaction volume, $\Delta \&$ is the solid angle defined by the collection geometry, $G$ measures the spatial overlap of the two crossed beams, and $T$ is the integrat!on time for each yield measurement. In the present work, relative cross sections are obtained by comparing, under identical geometrical conditions, the ylelds of electron produced in the photodetachment of the ions of interest, $Y(x)$, and a bean of reference ions, $Y(r)$, whose cross section is known. Thus the relative cross sectione for the two photodetachant procesees can be written an 


$$
\frac{\sigma(x)}{\sigma(r)}=\frac{Y(x) \rho(r) \phi(r) g(r)[1+\beta(r)]}{Y(r) \rho(x) \phi(x) g(x)[1+\beta(x)]} .
$$

Here the measured yield ratio is multiplied by several measured factors in order to account for the differen: frame-transformed solid angles, photon fluxes, ion densities, and asymmetry parameters associated with photodetaching electrons fror the two beams. All geometric factors, being equal, cancel out. In this work it is arranged by appropriate cholce of beam energies, that the photoelectron peaks in each spectra are kinematically shifted so that they colncide in energy in the laboratory frame. The efficlency factors, $k(x)$ and $k(r)$, associated with the collection and detection of photoelectron at the same energy from the lons of interest and the reference lons wlll then be equal and hence cancel out. For example, F1g. 6 shows three photoelectron spectra in wh1ch the electron peaks all occur at $-11 \mathrm{eV}$ in the laboratory frame. The top and bottom peaks are associated with the photodetachment of $\mathrm{He}^{-} \mathrm{via}$ the $\left.\mathrm{He}^{3} \mathrm{~S}\right)$ and $\mathrm{He}\left({ }^{3} \mathrm{P}\right)$ exit channels, respectively. The peak of the middle spectrum arises from the photodetachment of $D^{-}$ions in the reference beam via the $D\left({ }^{2} S\right)$ exit channel. To ensure that the electrons from these three different photodetachment channels are analyzed at the same energy in the laboratory frame, the Ion beam energies for the top, middle, and bottom spectra were chosen to be $29.19,19.17$, and $50.47 \mathrm{kev}$, respectively.

The lon beam densities, $p$, and the solidangle transformation factors, $g$, both depend on the velocity of the ion beam. This quantity can be determined very precisely $(\sim 0.1 \%)$, in situ, by simply analyzing the photoelectron spectra. In $\mathrm{Fig}_{\mathrm{g}}$ 4, the backward-directed peak $1\left(\theta_{c}=\pi\right)$ and the forward-directed peak $2\left(\theta_{c}=0\right)$ form a kinematically-doubled pair, both belng assoclated with the photodetachment process that leaves the He atom in the $2^{3} \mathrm{p}$ state. Their separation, in the laboratory frame, is $\Delta E_{L}(12)=4 \sqrt{\varepsilon E_{c}(I)}$. The quantity $E_{c}$, the energy of the electron in the lon frame, can be determined from a measurement of the photon wavelength and the use of the precise theoretical value of the electron affinity of $\mathrm{He}\left(2^{3} \mathrm{~S}\right)$ calculated by Bunge et a1.13 Thus by measuring $\triangle E_{L}(12)$, one can determine the reduced ion beam energy, $\varepsilon$, and hence the lon beam energy itself. Another way of determining the fon beam energy is by measuring the separations of peaks 2 and 3 . Peak 3 is due to the forward-directed electrons assoclated with the competing process $v 1$ a the he $\left(2^{3} S\right)$ exit channel. In the lon frame, peaks 2 and 3 are separated by the $2^{3} S-2{ }^{3} p$ transition energy, $E_{e}$, known precisely from optical spectroscopy. The separation of peaks 2 and 3 in the laboracory frame is given by

$$
\Delta E_{L}(23)=E_{e}+2 \sqrt{c}\left[\sqrt{E_{c}(2)+E_{e}}-\sqrt{E_{c}(2)}\right] \cdot(7)
$$

In a similar manner, the velocities of beams of other lons, such as $\mathrm{D}^{-}$and $\mathrm{B}^{-}$, can be prectsely determined by measuring the separation between the peaks of their photoelectron spectra and the peaks of the $\mathrm{He}^{-}$spectrum.

Angle-resolved measurements are made to determine the asymmetry parameter, $\beta$. The yield of a photoelectron peak is measured as a

Photodetechment Spectra ( $\lambda=696.2 \mathrm{~nm})$
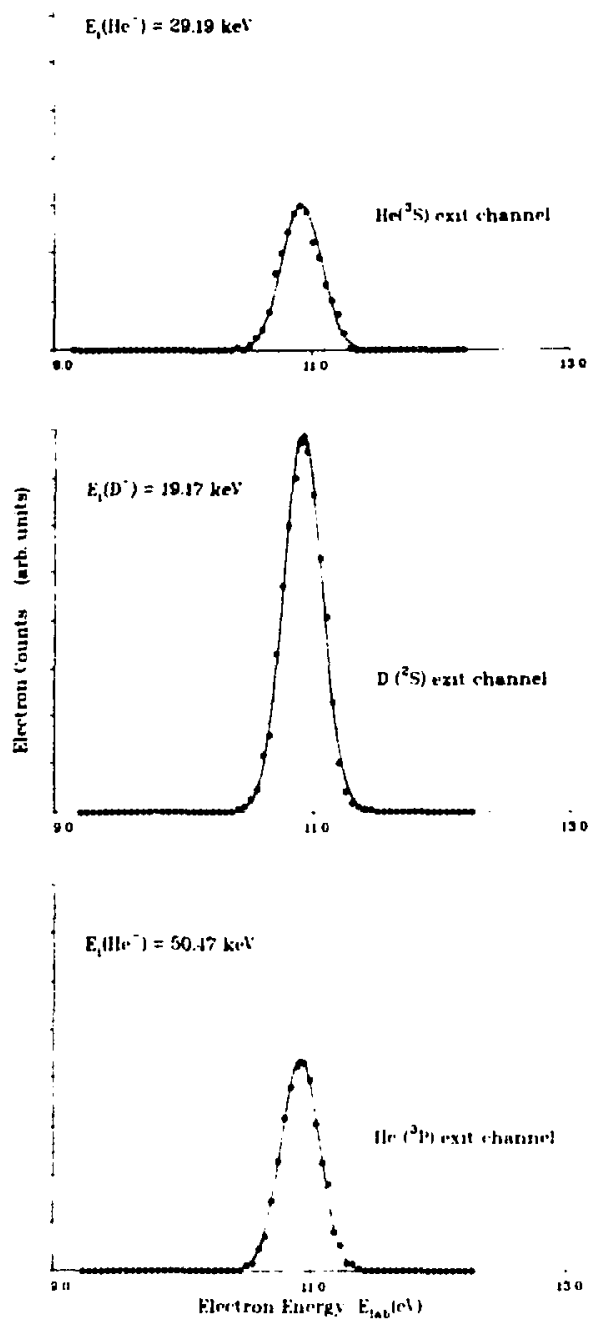

FIG. 6. Three spectral peaks kinematically shifted to the same laboratory-frame energy by an appropriate cholce of lon beam energy. The peak ylelds were used in the measurement of relative crose sections for photodetachment of He and De lons. 
sunction of the angle, $\theta$, between a fixed collection direction (in the present case, the direction of motion of the ion beam) and the variable direction defined by the electric field vector of the linearly polarized laser beam. For the asse of plane polartzed radiation in the electric dipole approximation (assuming an adependent electron model), the angular correlation between the outgoing photoelectron and the incoming photon should take the form, $f(e)=1+\beta P_{2}(\cos \theta)$. Here $P_{2}(\cos \theta)$ is the second-order Legendre polynomiai. Multiphoton absorption or an ion state that is prepared anisotropically prior to photodbsorption would produce higher (even) order polynomials in the fit. It inas been demonstrated that both processes akike a negligitle contribution in the present work. The angular distribution measurement technique has been tested, at several difterent wavelengths in the visible, by photodetaching beams of $\mathrm{D}^{-}$and $\mathrm{if}^{-}$ions. The predicted $\cos ^{2} \theta(\beta=2)$ distribution was obtafned is all cases.

Angular differential cross sections, $I_{c}(\theta)$, an nost easily be determined fron the measured angular integral cross sections, $\sigma$, and the asymetry parameters, $\beta$, using the expression

$$
I_{c}(\theta)=\frac{O}{4 \pi}\left[1+\beta P_{2}(\cos \theta) \mid\right. \text {. }
$$

\section{v. RESULTS}

\section{A. Partial Cross Sectlons for $\mathrm{He}^{-}$ Photodetachment}

The partial (angular integral) cross sections, $\sigma\left({ }^{3} S\right)$ and $o\left({ }^{3} \mathrm{P}\right)$, for photodetaching eleccrons from $\mathrm{He}^{-}\left(2^{4} \mathrm{P}\right)$ via the He$\left(2^{3} \mathrm{~S}\right)$ and the Ie $\left(2^{3} \mathrm{p}\right.$ ) exit channels, respectively, have been ineasured telative to the cross section, $\sigma\left({ }^{2} S\right)$, for photodetaching $D^{-}$via the $D\left(1^{2} S\right)$ exit channel. The ratio of the $\sigma\left({ }^{3} \mathrm{~S}\right)$ to $\sigma\left({ }^{3} \mathrm{P}\right)$ cross sections has also been measured. At a wavelengtl of $\lambda=696.2 \mathrm{~nm}$, the following results were obi.t ned: $\sigma\left({ }^{3} \mathrm{~S}\right) / \sigma\left({ }^{2} \mathrm{~S}\right)=0.60 \pm 0.02, \sigma\left({ }^{3} \mathrm{~S}\right) / \sigma\left({ }^{3} \mathrm{P}\right)=$ $2.28 \pm U .10$, and $\sigma\left({ }^{3} \mathrm{p}\right) / \sigma\left({ }^{2} S\right)=0.27 \pm 0.02$. The uncertainties quoted on these values are at the level of two standard deviations of the weighted mean obtained trom about ten data sets, each consisting of a number of individual spectra. The major source of statistical error in the ratios obtained from the individual data sets stems from neasurements of the photoelectron yield $(\sim 4 \%)$ and asymetry parameter $(\sim 3 \%)$ ratios. Uncertainties in the measurement of other factors in Eqn. (6) are negligtbly small. Systenatic errors are also estimated to be within the zuoted limits. The asymetry parameters characterizing the photoelectron angular distributions have also been seasured at $\lambda=696.2 \mathrm{am}$. The results are shown ir Table $I$ along with the angular fintegral cross section results. Ar absolute scale for the $\mathrm{He}^{-}$partial cross sections has been established by assuming a theoretical value for the $\sigma\left({ }^{2} S\right)$ criss section. The calculations of Stewart, 14 for example, which are estimated to have an uncertainty of better than $3 \%$, produce a result of $\sigma\left({ }^{2} \mathrm{~S}\right)=38.1 \mathrm{4b}$ at $\lambda=696.2 \mathrm{~nm}$. Broad and Reinharats calculate a value within $1 \%$ of this result. Combining the theoretical result (assuming a $3 \%$ uncertainty) with the measured ratios produces the partial (angular integral) cross sections shown in Table $\mathrm{I}$. Table $\mathrm{I}$ indicates that at a wavelength of $696.2 \mathrm{nn}$, the probability that a glven $\mathrm{He}^{-}$ion photodetaches lato the lle $\left(^{3} \mathrm{~S}\right)+\mathrm{e}^{-(\mathrm{ks}, \mathrm{c})}$ continuum is $70 \%$ (1.e. the branching ratin, $\left.R\left({ }^{3} S\right)=0.70 \pm 0.06\right)$. At the present $t \mathrm{ime}$, there are no other experimental or theoretical results to compare with these neasured partial cross sections. The sum of the partial cross sections, $\sigma$, can, however, be compared to the total cross section calculations of ilazi and Reed.16 The scatter plot shown in Fig. 7 indicates that there is excellent agreement between the present result of $\sigma=\sigma\left({ }^{3} S\right)+\sigma\left({ }^{3} P\right)=$ $32.9 \pm 2.4 \mathrm{Mb}$ and the theoretical result of $\sigma=33.4 \mathrm{Mb}$ (length form) and $0=29.9 \mathrm{Mb}$ (velocity form). Also shown in Fig. 7 are the experinental results of Compton et al..$^{7}$ and ilodges et a1.8 These less precise values are also seen to be in agreement with the present result.

The angular integral cross sections, $\sigma$, and the asymetry parameters, $\beta$, can be conveniently combined, using Eqn. (7) to determine the angulat differential cross sections, $I_{c}(\theta)$. The results are shown in $\mathrm{FIg}_{\mathrm{g}}$. 8 .

TABLE I. Measured Angular Integral Photodetachment Cross Sections and Asymmetry Parameters at $\lambda=696.2$ na.

\begin{tabular}{lll}
\hline & $\sigma(\mathrm{Mb})$ & $\beta$ \\
& & \\
$\mathrm{Hex}\left({ }^{3} \mathrm{~S}\right)$ & $22.9 \pm 1.0$ & $1.15 \pm 0.02$ \\
$\mathrm{He}\left({ }^{3} \mathrm{P}\right)$ & $10.0 \pm 0.6$ & $1.52 \pm 0.04$ \\
$\mathrm{~B}\left({ }^{2} \mathrm{P}\right)$ & $16.5 \pm 2.1$ & $0.18 \pm 0.04$ \\
$\mathrm{D}\left({ }^{2} \mathrm{~S}\right)$ & $38.1 \pm 1.1^{\mathrm{a}}$ & $2.00-0.02$
\end{tabular}

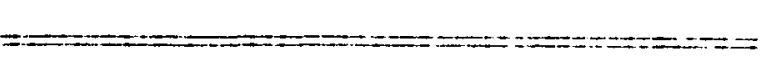

areference 14. 
Tutal Cross bection for !hotodetachment of Melastable $\mathrm{He}^{-}, \lambda=696.2 \mathrm{~nm}$

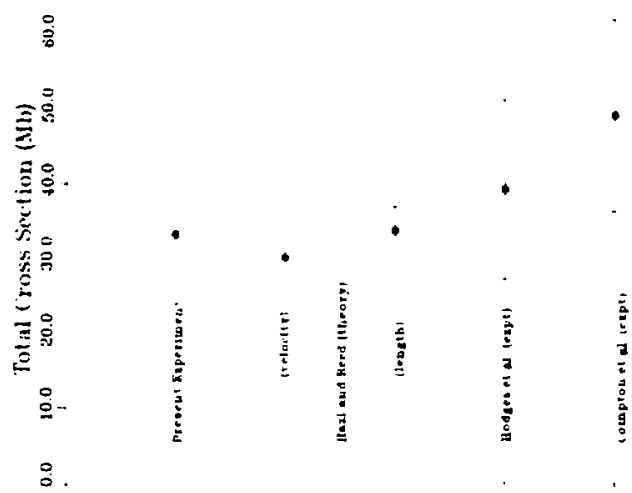

FIG. 7. Scater dlagram showing different experimental and theoretical results for the total (angular integral) cross section for photodetachment of the $\mathrm{Hle}^{-}$Ion at $\lambda=696.2 \mathrm{~nm}$.

Differential Cross Section for the

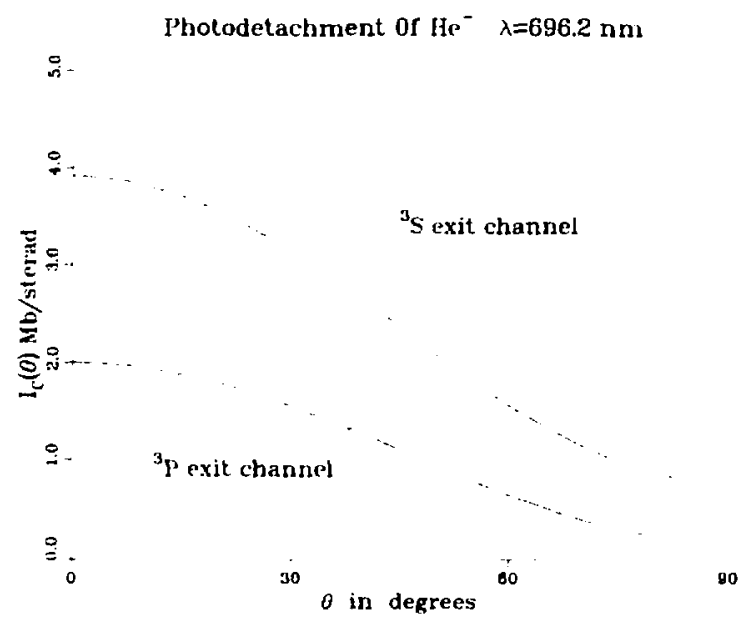

FIG. 8. Measured angular differential cross sections for the photodetachment of the $\mathrm{He}^{-}$ion at $\lambda=696.2 \mathrm{~nm}$.

\section{B. Cross Section for $B^{-}$Photodetachment}

The cross section, $\sigma\left({ }^{2} \mathrm{P}\right)$, for the photodetachment of $B^{-}$via the $B\left(2^{2} P\right)$ exit channel has been measured relative to the cross section, $a\left({ }^{3} \mathrm{P}\right)$, for the photodetachment of $\mathrm{He}^{-} \mathrm{vla}$ the He $\left(2^{3} \mathrm{P}\right)$ exit channel at a wavelength of $\lambda=$ $696.2 \mathrm{~nm}$. The $\mathrm{B}^{-}$and $\mathrm{He}^{-}$Ion beam energles were chosen to be 51.04 and $30.18 \mathrm{keV}$, respec- tively, so that the peaks in the photoelectron spectra coincided in energy in the laboratory frawe. This ensured that photoelectrons from the two beams were collected and detected with equal effictency. The result of the measurement is $\sigma\left({ }^{2} \mathrm{P}\right) / \sigma\left({ }^{3} \mathrm{P}\right)=1.65 \pm 0.19$. The asymmetry parameter for this process has been measured and the result is shown in Table I along with angular Integral cross section. In this cas?, an absolute scale for the $\sigma\left({ }^{2} \mathrm{P}\right)$ cross section has been established by assuming the value of the of $\left({ }^{3} \mathrm{P}\right)$ cross section that had previously been measured relative to the $\sigma\left({ }^{2} \mathrm{~S}\right)$ cross section of $\mathrm{D}^{-}$, 1.e. $\sigma\left({ }^{3} \mathrm{P}\right)=10.0 \pm 0.6 \mathrm{Mb}$. There are no other existing results, experimental or theoretical, to compare with the present measurement.

Again, the angular differential cross section, $I_{c}(\theta)$, is conventently determined from the measured angular integral cross section, $\sigma$, and the asymetry parameter, $\beta$. The results are shown in Fig. 9.

\section{Differential Cross Section for the} Pholodetachment of $B^{-} \quad \lambda=696.2 \mathrm{sm}$

ลิ-

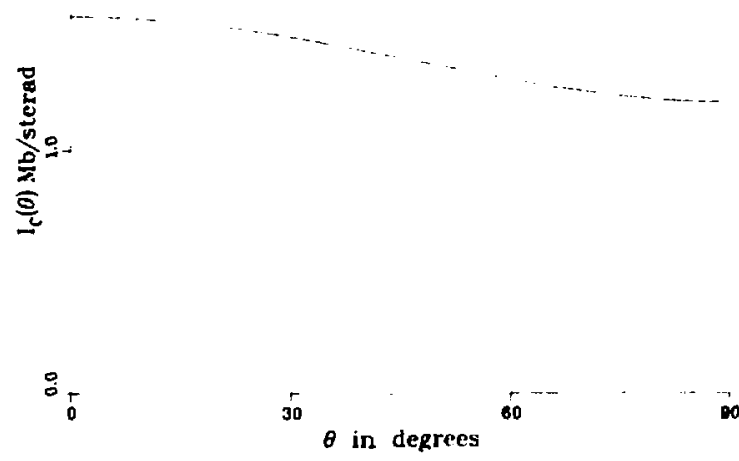

FIG. 9. Measured angular differential cross section for photodetaching the $B^{-}$Ion at $\lambda=696.2 \mathrm{rm}$.

\section{Radiative Attachment Cross Sections}

The measured cross sections for photodetaching $\mathrm{He}^{-}$and $\mathrm{B}^{-}$lons can be used to indirectly determine, using detalled balance arguments, the cross sections, $\sigma_{a}$, for the far more Improbable inverse process of radiative attachment. The processes are the Inverse of those shown in Eqns. (1), (2), and (3). The derived radiative attachment cross sections (in barns) at $\lambda=696.2$ tm are shown in Iable II. 
TABLE II. Derived Radlative Attachment Cross Sections at $\lambda=696.2 \mathrm{~nm}$.

\begin{tabular}{cc}
\hline & $\sigma_{\mathrm{a}}(\mathrm{b})$ \\
\hline Encrance Channel & $166 \pm 7$ \\
$\mathrm{He}\left({ }^{3} \mathrm{~S}\right)$ & $74.2 \pm 4.5$ \\
$\mathrm{He}\left({ }^{3} \mathrm{P}\right)$ & $51.0 \pm 6.5$ \\
$\mathrm{~B}\left({ }^{2} \mathrm{P}\right)$ & \\
\hline
\end{tabular}

VI. SUMMARY

A crossed-beams apparatus that employs energy- and angle-resolved photoelectron spectroscopy has been used to ineasure angular differential and integral cross sections for the photodecachment of $\mathrm{He}^{-}$and $\mathrm{B}^{-}$lons at a wavelength of $696.2 \mathrm{~nm}$. An absolute scale has been established by making relative photoelectron yield and angular distribution "measurements between beams of the lons of interest and $D^{-}$ reference Ions whose photodetachment cross section is known from theory to better than $3 \%$.

Absolute cross sections are difficult to determine accurately. The relatively high precision ( $5 \%$ in favorable cases) of the present measurements reflects our ability to exploit, in a novel way, certain kinematic effects associated with a fast moving source of lons and the collection of ejected photoelectrons in the forward direction. The measurements are currently being continued at several discrete wavelengths across the vistble spectrum. It is hoped that these new results will stimulate further calculations of photodetachment cross sections and asymmetry parameters.

\section{ACKNOWLEDGMENTS}

I wish to acknowledge my fellow collaborators, past and present, at the Unfversity of Tennessee (J.S. Thompson and J. Dellwo) and the Oak Ridge National Laboratory (G. D. Alton, R. N. Compton, and T. J. Kvale). Special thanks go to Jeff Thompson. This research was supported by the U.S. Department of Energy, office of Basic Energy Sclences, Division of Chemical Sciences chrough the University of Tennessee. Oak Ridge National Laboratory is operated by Martin Marietta Energy Systems, Inc. under Contruct No. DE-AC05-84OR21400 with the U.S. Department of Energy.

\section{REIERENCES}

1. K. Dolder, in Electronic and Atomic

Colissions (Invited papers of the XV ICPEAC), ed. by H. B. Gilbody, W. R. Newe11, F. H. Read, and A.C.H. Smith (North-Holland, Amsterdam, 1988), p.549.

2. L. M. Branscomb and W. L. Fite, Phys. Rev. 93, A651 (1954).

3. L. M. Branscomb, in Atomic and Molecular

Processes, ed. by D. Bates (Academlc, New York, 1962), p. 100 .

4. S. J. Smith, in Methods of Experimental

Physics, ed. by B. Bederson and W. Fite

(Academ1c, New York, 1968), Vol. 7, p. 179.

5. W. C. Lineberger, in Applied Atomic Collision Physics, ed. by H.S.W. Massey, E. W. McDaniel and B. Bederson (Academic, New York, 1982), Vol. 5, p. 239.

6. B. Brehm, M. A. Gusinow, and J. L. Hall, Phys. Rev. Lett. 19, 737 (1967).

7. R. N. Compton, G. D. Alton, and D. J. Pegg, J. Phys. B 13, L65I (1980).

8. R. V. Hodges, M. .1. Cogglola, and J. R. Peterson, Phys. Rev. 23, 59 (1981).

9. L. M. Blau, R. Novick, and D. Welnflash, Phys. Rev. Lett. 24, 1269 (1970).

10. J. S. Thompson, D. J. Pegg, R. N. Compton, and G. D. Alton, J. Phys. B (to be published).

11. D. J. Pegg, J. S. Thompson, R. N. Compton, and G. D. Alton, Phys. Rev. Lett. 59, 2267 (198\%).

12. D. J. Pegg, J. S. Thompson, R. N. Compton, and G. D. Alton, Nucl. Instrum. Meth. B 40/41, 221 (1989).

13. A. V. Bunge and C. F. Bunge, Phys, Rev. A 19, 452 (1979).

14. A. L. Stewart, J. Phys. B 11, 3851 (1978). 15. J. T. Broad and W. P. Reinhardt, Phys. Rev. A 14,2159 (1976).

16. A. U. Hazl and K. Reed, Phys. Rev. A 24, 2269 (1981). 